result in the DETERMINE ${ }^{\circledR}$ Syphilis test at follow-up. False positive tests with DETERMINE ${ }^{\circledR}$ Syphilis test were defined as being non-confirmed by combined cardiolipin and specific treponemal positive tests at baseline and negativity in the follow-up period.

Results Of all positive results with the DETERMINE ${ }^{\circledR}$ Syphilis test $14.0 \%$ were shown to be true positive results by fulfilling one or more of the aforementioned criteria, compared to combined conventional tests. Compared to the use of single cardiolipin tests, the DETERMINE $^{\circledR}$ test detected $27.1 \%$ more cases. False positive tests with DETERMINE Syphilis TP test occurred $0.26 \%$ of those patients tested.

Conclusions The DETERMINE ${ }^{\circledR}$ syphilis test is superior to treponemal and cardiolipin tests alone or in combination in the detection of syphilis in Primary Care.

\section{P2.072 SEEK AND YOU SHALL FIND - VALUE OF EXTRAGENITAL CHLAMYDIA AND GONORRHOEA TMA TESTING IN A COHORT OF MSM}

doi:10.1136/sextrans-2013-051184.0337

J W Shaw, S Ahmad. University Hospital of South Manchester, Manchester, UK

Background TMA (Aptima Combo II) is unlicensed for the detection of chlamydia and gonorrhoea at extragenital sites but is increasingly used to screen MSM reporting receptive oral or anal intercourse as data accumulates to support its performance. We reviewed its value in screening our MSM cohort.

Methods The TMA and culture results of MSM receiving extragenital screening between 01/01/12 and 30/06/12 were retrospectively reviewed.

Results 565 MSM were screened (1042 extra-genital samples); 95 tested positive for at least one infection at one site.

111 positive TMAs were extragenital (detection rate 10.7\%) amongst 87 patients. 68 patients had isolated extragenital infection.

$41 \mathrm{MSM}(8.5 \%)$ had rectal chlamydia, only 5 of whom had genital chlamydia; 7 were confirmed LGV.

$8(1.4 \%)$ MSM had pharyngeal chlamydia. 4 had concurrent rectal infection; none had urethral infection.

Urethral chlamydia was detected in 16 MSM (2.8\%).

27 (5.6\%) MSM had rectal gonorrhoea via TMA; 11 were positive on culture.

$35(6.2 \%)$ MSM had pharyngeal gonorrhoea via TMA; 8 were positive on culture.

$12(2.1 \%)$ MSM had urethral gonorrhoea; 11 were positive on culture.

Conclusion $15.4 \%$ MSM attending for tests had extragenital infection. $71.6 \%$ testing positive had isolated extragenital infection Without extragenital TMA tests 49 chlamydia infections would have been missed or suboptimally treated, including 7 LGV.

$25 / 52$ cases of gonorrhoea would have remained undiagnosed had screening been by 3 site culture and urethral TMA.

Undiagnosed extragenital infection has implications regarding onward transmission of that STI and may result in missed/suboptimal treatment and partner notification. Failure to screen effectively may provide false reassurance and reinforce popular myths that the pharynx is not vulnerable to infection. This data supports our current practise of TMA testing at extragenital sites.

\section{P2.073 EXTRAGENITAL SCREENING IN WOMEN - IS TMA VALUE FOR MONEY?}

doi:10.1136/sextrans-2013-051184.0338

J W Shaw, S Ahmad. University Hospital of South Manchester, Manchester, UK

Background Transcription mediated amplification (TMA, Aptima Combo II) is currently unlicensed for the detection of chlamydia and gonorrhoea at extragenital sites. Accumulating evidence suggests TMA has high sensitivity and specificity for pharyngeal and rectal infection. We have offered pharyngeal and rectal TMAs to all patients presenting since April 2009 reporting receptive oral or anal intercourse. Methods The TMA and culture results of heterosexual women receiving extragenital screening between 01/01/12 and 30/06/12 were retrospectively reviewed.

Results 1315 women were screened (1520 extra-genital samples), 79 tested positive for at least one infection at one site. $62(4.7 \%)$ patients had genital chlamydia. $9(0.7 \%)$ patients had genital gonorrhoea.

33 positive results were extragenital (detection rate $2.17 \%$ ) with 10 patients having isolated extragenital infection.

Rectal chlamydia detection rate was 3.8\%. Of the 8 patients with rectal chlamydia, 7 had co-existing genital infection.

$14 / 1309$ (1.1\%) patients had pharyngeal chlamydia, 5 had isolated pharyngeal infection.

$6 / 211(2.8 \%)$ patients had rectal gonorrhoea, 1 had isolated rectal infection.

$5 / 1309(0.4 \%)$ patients had pharyngeal gonorrhoea, 3 had isolated pharyngeal infection.

There were no positive extragenital gonorrhoea cultures and no cases of extragenital dual infection.

Conclusion Detection rates for extragenital chlamydia (both sites) and rectal gonorrhoea exceeded that of genital gonorrhoea.

Without extragenital screening we would have failed to treat 10 women with isolated pharyngeal or rectal infection, i.e. $12.7 \%$ of all women testing positive.

Regarding the 8 women with rectal chlamydia, 7 could have been suboptimally treated with azithromycin and one would have been missed.

This has implications for onward transmission, enhanced transmission of other STIs, and missed opportunities for partner notification.

Failure to screen women extragenitally may reinforce the misconception that these sites are not as significant in STI transmission and encourage risk taking behaviour.

\section{P2.074 COMPARISON OF SPECIMEN TYPE FOR THE DIAGNOSIS OF TRICHOMONAS VAGINALIS (TV) USING THE VIPER ${ }^{\text {TM }}$ SYSTEM IS EXTRACTED MODE}

doi:10.1136/sextrans-2013-051184.0339

J R Schwebke, ${ }^{2} \mathrm{D}$ Fuller, ${ }^{3} \mathrm{~S}$ Taylor, ${ }^{4} \mathrm{~J}$ Lebed, ${ }^{5} \mathrm{~B}$ Smith, ${ }^{6} \mathrm{~B}$ Van Der Pol, ${ }^{7} \mathrm{M}$ Nye, ${ }^{1} \mathrm{C}$ Rivers, ${ }^{1} \mathrm{C}$ Aycock, ${ }^{8} \mathrm{C}$ Gaydos. ${ }^{1}$ University of Alabama at Birmingham, Birmingham, AL, United States; ${ }^{2}$ Wishard Health Services Indiana School of Medicine, Indianapolis, IN, United States; ' LSU Health Sciences Center, New Orleans, LA, United States, ${ }^{4}$ Planned Parenthood Southeastern PA, Philadelphia, PA, United States; ${ }^{5}$ Planned Parenthood of Gulf Coast Inc, Houston, TX, United States; 'Indiana University School of Medicine, Indianapolis, IN, United States; ' LabCorp, Burlington, NC, United States; ${ }^{8}$ The Johns Hopkins University, Baltimore, MD, United States

Background TV is the most common curable STI worldwide and is associated with adverse consequences including preterm birth and acquisition/transmission of HIV. Yet, until recently, testing options for this infection were limited and had suboptimal sensitivity. Recently, nucleic acid amplification test systems (NAATS) for diagnosis of TV have been developed. Various types of specimens were studied in this process. We now report on the agreement of these specimen types for the diagnosis of TV using this NAATS assay.

Methods Eight centres participated in this study. Specimens were collected from subjects presenting with symptoms of trichomoniasis or for routine visits. Specimens were collected in the following order: (1) first void urine, (2) patient-collected vaginal swab, (3) three clinician-collected vaginal swabs, (4) endocervical swab. Urine was aliquotted into a Viper neat and UPT tube for BD Probe Tec ${ }^{\text {TM }}$ Trichomonas vaginalis (TV) $\mathrm{Q}^{\mathrm{x}}$ Amplified DNA Assay (TVQ) testing. The three clinician-collected vaginal swabs were used for wet mount, culture, and comparator testing. Sensitivity and specificity for the specimen 